\title{
MANIFESTATIONS OF TWO-DIMENSIONAL ELECTRON GAS IN MOLECULAR CRYSTALS
}

\author{
Maija M. Kuklja ${ }^{1}$, Onise Sharia, and Roman Tsyshevsky
}

\begin{abstract}
The existence of two-dimensional electron gas in molecular materials has not been reported or discussed. Intriguing properties of two-dimensional electron gas observed on interfaces of polar and nonpolar oxides spurred oxide electronics and advanced nanotechnology. Here we discover how an electrostatic instability occurs on polar surfaces of molecular crystals and explore its manifestations, chemical degradation of surfaces, charge separation, electrical conductivity, optical band-gap closure and surface metallization. A thin layer of polar surface of a dielectric molecular crystal becomes metallic due to interactions of polar molecules. Our findings are illustrated with two polymorphs of cyclotetramethylenetetranitramine crystals, the polar $\delta$-phase and nonpolar $\beta$-phase. Our theory offers an explanation to a relative stability of the $\beta$-phase versus the explosive reactivity of $\delta$-phase and to the experimentally observed difference in conductivity of these crystals. We predict that the electrostatic instability takes place on all polar molecular materials.
\end{abstract}




\section{Introduction}

Two-dimensional electron gas observed on interfaces of polar and nonpolar oxide surfaces $\left[{ }^{1,2,3}\right]$ led to revealing exotic phases of matter $\left[{ }^{4,5}\right]$ that exhibit metal-insulator transitions $\left[{ }^{6,7}\right]$, superconductivity $\left[{ }^{8,9}\right]$ and its controllable on/off switching $\left[{ }^{10}\right]$, large negative magnetoresistance $\left[{ }^{11}\right]$, co-existence of superconducting and magnetic states $\left[{ }^{12}\right]$, and giant thermoelectric effect $\left[{ }^{13}\right]$. Those unusual properties prompted the emerging field of oxide electronics $\left[{ }^{14}\right]$ and opened new prospects in nanotechnology $\left[{ }^{15}\right]$ among other intriguing opportunities. However, the existence and properties of two-dimensional gas in molecular materials have yet to be established. Here we discover an electrostatic instability that generates the two-dimensional electron gas state and takes place on polar surfaces in molecular crystals. A thin layer of polar surface of a wide band gap dielectric molecular crystal becomes metallic due to interactions of polar molecules. Among fundamental manifestations of this phenomenon, we theoretically explore chemical degradation of surfaces, charge separation, electrical conductivity, optical band-gap closure and surface metallization. We found that there is a dramatic difference in surface decomposition reactions of polar $\delta$ phase and nonpolar $\beta$-phase polymorphs of molecular cyclotetramethylene-tetranitramine (HMX) crystals (Figure 1). This difference is governed by excess or deficit of electrons, or surface conductivity, which explains a relative stability of the $\beta$-phase (Figure 1a-c) and the high reactivity of the $\delta$-phase (Figure 1d-f). The polarization-induced separation of electrons and holes on $\delta$-HMX creates the surface charge density of $7.9 * 10^{13} \mathrm{e} / \mathrm{cm}^{2}$, corresponding to 0.458 electrons/molecule, which is similar to a typical charge carrier density at the oxide/oxide interfaces. 


\section{Computational approach}

All molecular calculations in our study were carried out within the GAUSSIAN 09 [ ${ }^{16}$ ] program suite. Density functional theory $\left[{ }^{17,18}\right]$ (DFT) - based Heyd-Scuseria-Ernzerhof functional HSE06 $\left[{ }^{19}\right]$ with $6-31+\mathrm{G}(\mathrm{d}, \mathrm{p})$ basis set was used to study the electronic structure of ground state equilibrium molecules. Optical absorption properties were explored with the time dependent DFT (TD HSE06) procedure $\left[^{20,21}\right]$.

Periodic solid-state calculations were performed using the GGA PBE functional and PAW pseudo-potentials $\left[{ }^{22}\right]$ as implemented in the plane wave VASP code $\left[{ }^{23,24,25}\right]$. Details of calculations of ideal crystals of $\beta$ - and $\delta$-HMX are reported in $\left[{ }^{26,27}\right]$.

The structure of ideal bulk crystal of the hexagonal $\delta$-HMX (Figure 1e) was investigated by constructing a supercell consisting of 6 molecules (168 atoms) with lattice parameters of $a=b=8.18$ and $c=33.15 \AA$. In calculations of the ideal $\delta$-HMX crystal with the P6 $6_{1}$ symmetry, we used $2 \times 2 \times 1$ Monkhorst-Pack k-point mesh, and the kinetic energy cut-off was set to 600 $\mathrm{eV}$ (see ref. $\left[{ }^{26}\right]$ for more details).

Electronic properties of the $\delta$-HMX (001) surface were studied with the supercell model containing two symmetric lattice fragments each consisting of ten-molecule-thick (001) slabs (Fig. 2). Each ten-molecule fragment was cut out of the initially optimized hexagonal $\delta$-HMX bulk supercell (Figure 1e). Two crystalline pieces are placed in the supercell in such a way that one fragment in the slab represents a mirror reflection of the other fragment. The resulting supercell slab has parameters of $a=b=8.18 \AA, c=132.27 \AA$ and $\gamma=120^{\circ}$. The vacuum layer of $\sim 10 \AA$ placed on top of the $\delta$-HMX (001) surface was intended to minimize interactions between supercells in (001) direction and to ensure that electronic states of different slabs do not overlap. Such a 20-molecule (560-atom) supercell with zero total dipole moment was deliveraty designed to remove an artificial electric field in the vacuum layer. The full optimization of the surface structure was carried out using $\Gamma$-point only and the kinetic energy cut-off of $520 \mathrm{eV}$. A single point calculation using 6x6x1 Monkhorst-Pack $k$-point mesh was then carried out to obtain accurate density of states.

Electronic properties of the $\beta$-HMX (100) surface (Fig. 1c) were studied with the supercell consisting of nine molecular layers cut out of the optimized bulk crystal in the (100) direction (Fig. S1 of Supplementary Information). The resulting supercell contained 18 molecules (504 
atoms) and had lattice parameters of $a=62.31 \AA, b=11.41 \AA, c=8.91 \AA, \alpha=\beta=\gamma=90^{\circ}$. The thickness of the vacuum layer placed on top of the $\beta$-HMX (100) surface was of $\sim 10 \AA$. The full optimization of the surface structure was carried out using $\Gamma$-point only and the kinetic energy cut-off of $520 \mathrm{eV}$. A single point calculation using 6x6x1 Monkhorst-Pack $k$-point mesh was then carried out.

To correct significantly underestimated band gap energies, obtained from GGA PBE, a self-consistent single point calculation for each configuration was performed with the hybrid HSE06 functional at the $\Gamma$-point only and the kinetic energy cut-off set to $520 \mathrm{eV}$. A series of test calculations shows that such an approach yields reasonable band gap energies (See discussion in Supplemntary Information). In simulating optical absorption spectrum, we plotted the frequency-dependent imaginary part of the dielectric functions of ideal crystals and surfaces of $\beta$ - and $\delta$-HMX $\left[{ }^{28}\right]$.

Bader charges were analyzed by using Bader Charge Analysis code $\left.{ }^{29,30,31}\right]$. Coordinates of all structures employed in this study are collected in Supplementary Information.

\section{Results and Discussion}

The presence of a macroscopic surface dipole in oxide crystals is known to lead to an electrostatic instability, which can only be cancelled by the introduction of compensating charges in the outer planes $\left[{ }^{32}\right]$. This can be achieved either by a deep modification of the surface electronic structure - full or partial filling of surface states, sometimes leading to surface metallization $\left[{ }^{33,34,35,36}\right]$ - or by strong changes in the surface stoichiometry spontaneous desorption of atoms, faceting, large-cell reconstructions due to the ordering of surface vacancies, etc $\left[{ }^{37,38}\right]$.

We report an existence of two-dimensional electron gas in molecular crystals, as illustrated with a model example of two polymorphs of the organic molecular crystal of HMX with identical chemical compositions of $\mathrm{C}_{4} \mathrm{H}_{8} \mathrm{~N}_{8} \mathrm{O}_{8}$ and similar stoichiometry but different crystalline structures. HMX is an energetic material, often used as an explosive, fuel, and propellant. With four known HMX polymorphs, it is typically synthesized in its most stable at room temperature monoclinic $\beta$-phase (Fig. $1 \mathrm{a}-\mathrm{c}$ ) $\left[{ }^{39}\right]$, which, when heated to temperatures above $435 \mathrm{~K}$, converts to hexagonal $\delta$-HMX ${ }^{40}$, (Fig. $1 \mathrm{~d}$-f), the most chemically reactive phase. The transformation involves major changes of both the HMX crystal lattice from the 
monoclinic $\beta$-phase structure with the $P 2_{1} / c$ space group to the hexagonal $\delta$-phase structure with the space group $\mathrm{P}_{1}$ (Fig. $1 \mathrm{~b}$ and e) and the molecular ring conformation from $\beta$-chair (Fig. 1a) to $\delta$-boat (Fig. 1d) $\left[{ }^{39,40,41}\right]$.

Clearly, during the crystal growth and during the phase transition from the $\beta$-phase, the equilibrium shape of the $\delta$-HMX crystals will be determined assuming minimal surface free energy ${ }^{42,43,44}$ and certain crystal planes will be preferred over others ${ }^{45}$. The crystalline arrangement of the $\beta$-phase (Fig. 1b), which is composed on nonpolar HMX molecules (Fig. 1a), demonstrates that all possible free surfaces will be nonpolar. We chose a low energy (100) surface for our study (Fig. 1c). In contrast, the crystal structure of the $\delta$-phase (Fig. 1e), composed of polar molecules (Fig. 1d), suggests that even low energy surfaces will be polar, similarly to the (001) surface (Fig. 1f). Regardless of the crystal's shape, there always will be a facet made up from polar molecules, thus polar discontinuity cannot be avoided. In this work, we use the (001) surface as the effect is most prominent in this direction.

There are many experimental reports declaring a high chemical sensitivity of the $\delta$-phase to external thermal or mechanical perturbation, however the detailed reasons behind the dramatic differences in behavior of the HMX polymorphs has been remaining puzzling for decades $\left[{ }^{46}\right]$. The recent quantum-chemical study of decomposition reactions in the two crystalline phases suggested that the surface chemistry in the polar $\delta$-phase develops significantly faster than in the nonpolar $\beta$-phase $\left[{ }^{26,47}\right]$. This conclusion is consistent with the experimental observation that $\delta$-HMX is a more hazardous material and requires lesser external stimuli than $\beta$-HMX to reach detonation, even if their initial temperatures were the same $\left[{ }^{46}\right]$.

In unraveling intriguing physics behind the significantly different surface chemistry of those molecular crystals, we show that the explosive degradation of polar surfaces is an appearance of the electrostatic instability, which is largely unexplored in molecular materials. 

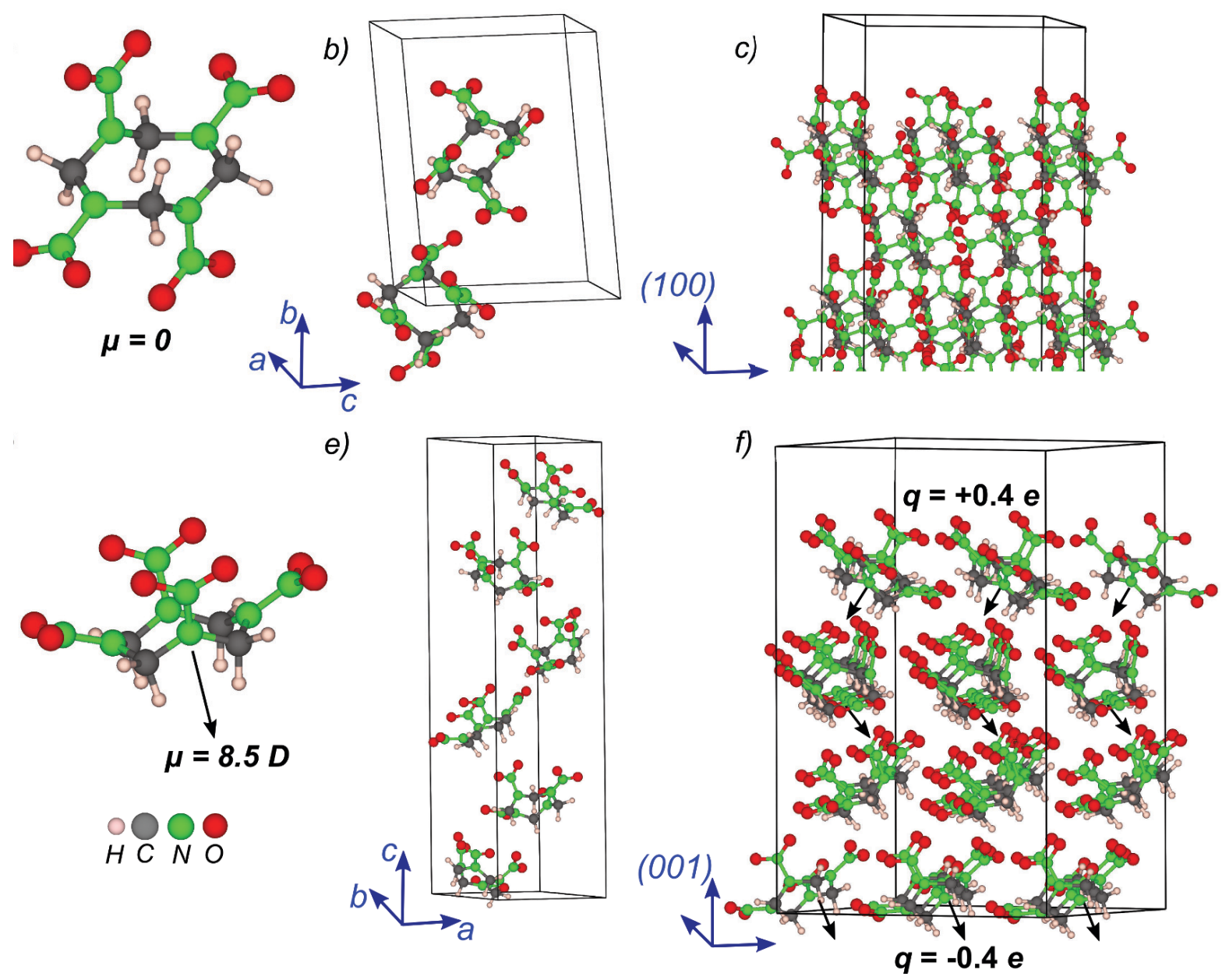

Fig. 1. The structure of a) $\beta$-HMX molecule, b) $\beta$-HMX crystal, c) nonpolar (100) $\beta$-HMX surface, d) $\delta$-HMX molecule, e) $\delta$-HMX crystal, and f) polar (001) $\delta$-HMX surface.

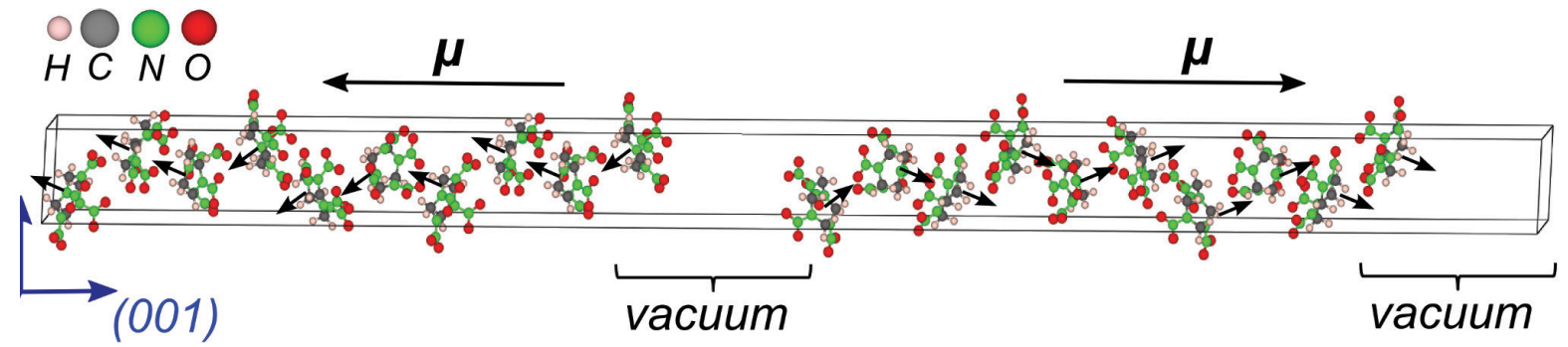

Fig. 2. The slab model of the ten-molecule-layer $\delta$-HMX (001) surface supercell. The model with two mirrored molecular crystal images separated by the vacuum layer is chosen to compensate for the dipole moment and to eliminate the electrostatic field. 
To introduce the polar catastrophe phenomenon for molecular crystals, consider a heterostructure with a discontinuity of polarization at the boundary between two materials. The interface between two different mediums has to satisfy the boundary condition $\left[{ }^{48}\right]$ :

$$
D 1-D 2 \cdot n=\sigma
$$

Here $D_{l}$ and $D_{2}$ are electric displacements, $D i=\varepsilon O i+P i, i$ is the electric field in the medium, and $P i$ is the polarization. The polarization can be written as a sum of an intrinsic polarization $P_{0}$ and an induced polarization $\Delta P=\chi$, which gives the displacement vector $D i=\varepsilon O \varepsilon i+P O$. Here, we consider the interface with vacuum (what is a free surface of a molecular material), therefore $D_{2}=0$. In the absence of a surface charge, eq. (1) dictates the presence of the electric field in the crystal:

$$
\perp=P O \cdot n \varepsilon O \varepsilon
$$

where $\perp$ is the normal component of the electric field. In macroscopic crystals, the electric field has to vanish otherwise the electrostatic potential will diverge with the size of the crystal. To avoid this electrostatic catastrophe, the surface (or interface) has to be charged with the surface charge density:

$$
\sigma=P O \cdot n
$$

Unlike ionic crystals $\left[{ }^{49,50,51,52}\right]$, molecular materials are constituted of molecules that tend to preserve their "identities" due to electronic functions well localized on the molecules. If the molecules are well separated, one can define molecular charge and dipole, and consequently define crystal polarization as:

$$
P O=1 \mathrm{Vipi}
$$

where $p_{i}$ is the dipole of $i$ molecule in a unit cell and $\mathrm{V}$ is the volume of the unit cell. Polarization defined by eq. (4) doesn't depend on the choice of the unit cell.

To explore surface properties and processes in real materials, an organic molecular crystal of HMX was selected as an ideal model system because it has polar ( $\delta$-phase) and nonpolar ( $\beta$-phase) polymorphs, which can be carefully analyzed from first principles. Figure 1f shows the structure of $\delta$-HMX with the identified (001) polar direction. For practical application of 
equation (4), one has to define boundaries between molecules. If molecules are very close to each other it is hard to define such boundaries. In case of $\delta$-HMX (Fig. 1f), boundaries between layers are clearly visible. Therefore $P_{z}$ can be calculated unambiguously using equation (4). For a formal definition of boundaries we use Bader method. We calculate the Bader volumes of individual atoms in a single layer and add them to obtain Bader volume of a layer. Within the Bader volume, the calculated $z$ component of the dipole per molecule is 2.54 eÅ. By using this theoretical value and the unit cell volume, $1921 \AA^{3}$, the polarization of $\delta$ HMX obtained from eq. (4) is $0.0079 \mathrm{e} / \AA^{2}$.

The Bader charges revealed the presence of positive and negative charges localized on the molecules placed on the surface layers of $\delta$-HMX (Fig. 1f), whereas the molecules located in the middle of the slab remain neutral. Our calculations of the surface slabs that were constructed of the gradually increased number of molecular layers (from one to ten), the surface charge approaches saturation at $0.4 e$ per molecule in the thickest ten layer slab (Fig. $3 a)$, which is in good agreement with the macroscopic limit of $0.458 e /$ molecule calculated from eq. (3). (This correlates with the reduction of the induced electric field, wich reaches 0.6 $\mathrm{eV} / \AA$ for the thin two-molecule-layer slab and nearly vanishes for the eight-molecule-layer slab (Fig. 3b)). We note that the corresponding surface charge density is $7.9 * 10^{13} \mathrm{e} / \mathrm{cm}^{2}$, which is similar to a typical value of the charge carrier density at the oxide/oxide interfaces (for example, $2 * 10^{13} \mathrm{e} / \mathrm{cm}^{2}$ is reported for LAO/STO interface $\left[{ }^{53}\right]$ ). By no means, this correspondence is accidental. It lends an additional support to the notion of the polarization generated charge and evidences that we are dealing, indeed, with the polar catastrophe in molecular materials. 

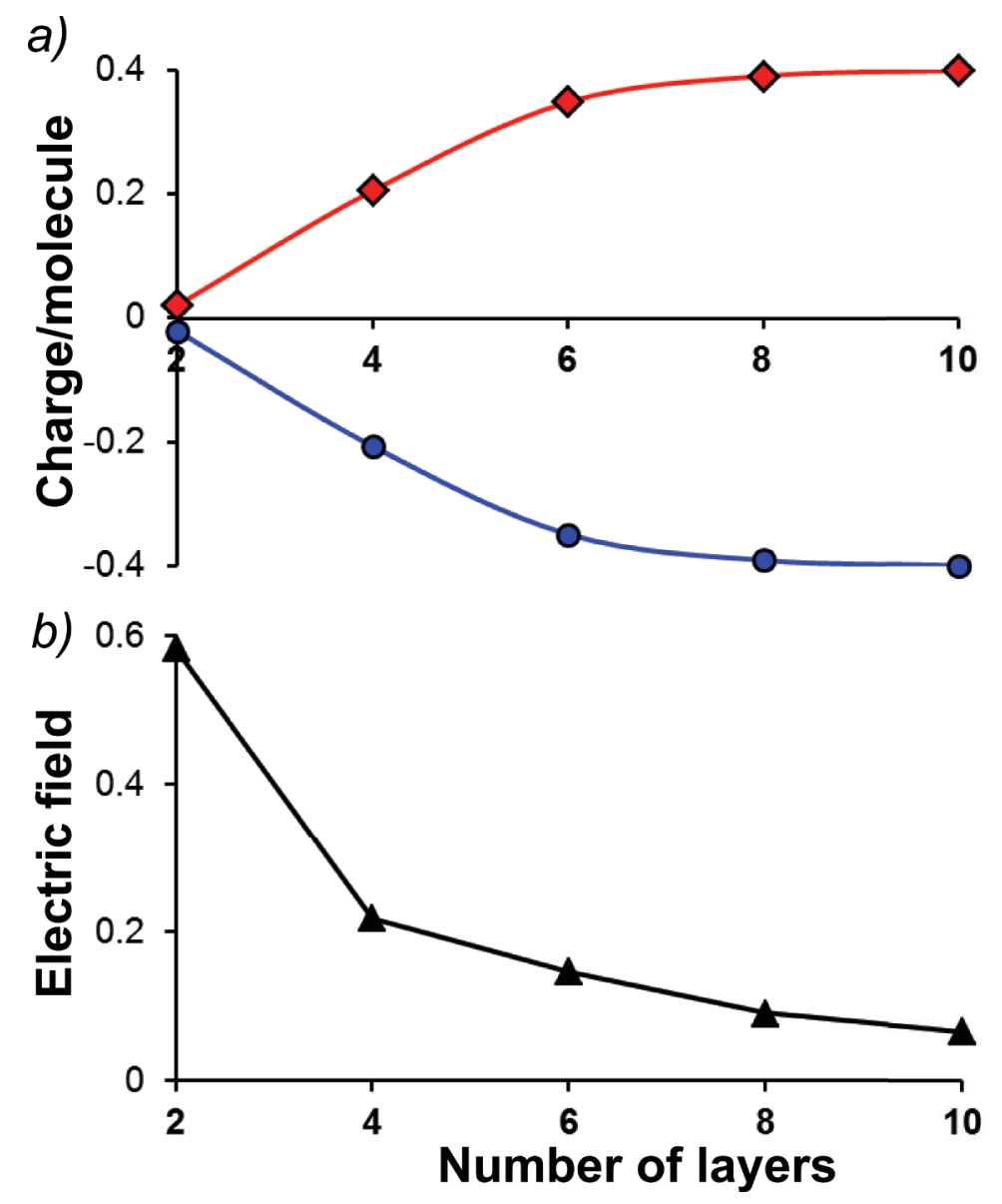

Figure 3. a) The charge of a surface molecule (in e/molecule) and b) the bulk electric field $(\mathrm{eV} / \AA)$ as a function of the number of molecular layers in the supercell slab model.

The redistribution of the charge density due to polarity of the material has a tremendous effect on the electronic structure, optical properties, and chemical reactivity of the material.

The effect of the surface charges on the electronic structure of the HMX surface was investigated by projecting density of states (DOS) on selected individual atoms (Fig. 4). The DOS projected on oxygen and nitrogen atoms of the $\delta$-HMX molecule placed in the ideal bulk (Fig. 4a), in the middle of the ten-layer model slab (Fig. 4b), on the positively charged surface (Fig. 4c), and the negatively charged surface (Fig. 4d) is displayed in Fig. 4. Fig. 4a and b show that bulk $\delta$-HMX is a wide gap insulator with the energy gap of $5.4 \mathrm{eV}$. This implies that the behavior of the molecules in the middle layer of the supercell slab replicates the performance of the molecules in the ideal bulk crystal. The top of the valence band (VB) is formed from $2 p$ functions of oxygen atoms of molecules placed on the positively charged 
surface and the bottom of the conduction band (CB) is formed from the $2 p$ functions of oxygen and nitrogen atoms of the molecule from the negatively charged surface (Fig. 4a). The surface electronic configuration is "metallic". The Fermi level intersects both the surface conduction band (Fig. 4c) and the valence band (Fig. 4d) composed by functions of molecules placed on the positively and negatively charged outer layers.

For comparison, the DOS projected on the oxygen and nitrogen atoms of the $\beta$-HMX molecule placed in the ideal bulk (Fig. 4 e), in the middle of the nine-layer model slab (Fig. $4 \mathrm{f}$ and Fig. S1 of Supplementary Information), and on the outer layers of the model slab (Fig. $4 \mathrm{~g}$ and h) also reveal the surface effect on the electronic structure of $\beta$-HMX, which is however significantly different. The calculated energy gap of bulk $\beta$-HMX is $5.1 \mathrm{eV}$, slightly smaller but consistent with $5.4 \mathrm{eV}$ corresponding to bulk $\delta$-HMX. The energy band gap of the $\beta$-HMX (100) surface reduces to $4.7 \mathrm{eV}$. The overall DOS projected on the molecule at the middle slab layer (Fig. $4 \mathrm{f}$ ) and on the outer surface molecules (Fig. $4 \mathrm{~g}$ and $\mathrm{h}$ ) show patterns similar to bulk (Fig. 4 e).

The calculated projected DOS leads us to conclude that the bulk $\delta$-HMX crystal, similarly to $\beta$-HMX, is a dielectric while the surface layers of $\delta$-HMX crystal, in contrast to $\beta$-HMX, behave as metallic conductors.

Experimental conductivity of HMX, $\sigma$, measured as a function of the activation energy, $E$, and temperature, and fitted to the standard equation $\sigma=\sigma 0 \exp -E 2 k B T$, determined that the activation energy of $\beta$-HMX is $5.2 \mathrm{eV}$ (approximately the band gap, $5.1 \mathrm{eV}$ ) and that of $\delta$ HMX is $2.9 \mathrm{eV}$, significantly lower than that of $\beta-\mathrm{HMX}\left[{ }^{54}\right]$. Measurements were performed on single crystals of $\beta$-HMX and polycrystalline samples of $\delta$-HMX and indicated that the $\beta$ phase conductivity is of the right order as organic solids having eight $\pi$ electrons expect to show activation energy $>4 \mathrm{eV}$ while the $\delta$-phase value is apparently unusually low $\left[{ }^{54}\right]$. Our proposed polarization discontinuity picture offers a clear and consistent interpretation to this phenomenon and explains the difference in phase conductivity registered in the experiment. The band structures of both phases of HMX are nearly identical for the bulk crystals and hence the abnormally high conductivity of $\delta$-HMX has to be related to solid-state effects, i.e. caused by a combination of the crystal morphology and uniqueness of defects in this phase.

In polar molecular crystals, the polar discontinuity arises near molecular defects and not only at the surface. Most common defects in molecular materials are vacancies and voids. 
Both of them can be considered as internal surfaces of a small radius $\left[{ }^{27}\right]$ and therefore should exhibit alike features as free surfaces. Indeed, an individual molecular vacancy created in $\delta$ HMX will exhibit a polar discontinuity at the vacancy site, which, on the other hand, will induce the local electric field. The electric field will shift energy levels of the molecules as it happens for the surface. However, the small size of the molecular vacancy causes only a local band gap reduction and no free carriers are generated. We observe such a band gap reduction for molecular vacancies in the bulk $\delta$-HMX but not in $\beta$-HMX.

A molecular vacancy in $\beta$-HMX was modeled by removing one molecule from a 16molecule supercell (Fig. S3 a) with the cell parameters of $a=13.47 \AA, b=22.82 \AA, c=17.81 \AA$, $\alpha=\gamma=90^{\circ}, \beta=124.1^{\circ}$. The supercell containing a single defect was built by a symmetric $2 \times 2 \times 2$ expansion of the $\beta$-HMX unit cell. The distribution of defects through the crystal corresponds to a $6.25 \%$ concentration of vacancies in the material. The band gap of the $\beta$-HMX crystal with vacancies uniformly distributed in the material is reduced relatively to the ideal model bulk crystal but this reduction does not exceed 3\%.

Isolated and bi-molecular vacancies in $\delta$-HMX were simulated in a 24 -molecule $\delta$-HMX supercell (Fig. S3 b) with the cell parameters of $a=16.36 \AA, b=14.168 \AA, c=33.152 \AA$, $\alpha=\beta=\gamma=90^{\circ}$. The supercell was constructed from the hexagonal $\delta$-HMX unit cell by choosing modified lattice vectors: $a=2(a O+b O), b=-a O+b O$, and $c=c O$. Such a supercell containing either single or bi-molecular vacancies mimics $4.17 \%$ and $8.34 \%$ concentration of vacancies in the crystal. Unlike the $\beta$-HMX crystal, the presence of isolated vacancies in $\delta$-HMX narrows the band gap by $\sim 28 \%$, and the bi-vacancies reduce it by $38 \%$. Hence, the vacancies affect the $\delta$-phase much stronger than the $\beta$-phase. More details on vacancy simulaions are provided in Supplementary Information.

Thus, the defects in $\delta$-HMX can cause two effects: a generation of a free charge and a reduction of the band gap. In both cases the electrical conductivity is increased. In the first case, the free carrier density is increased, which proportionally enhances the conductivity. In the second case, when the band gap is reduced, the activation barrier for conductivity is reduced. Once we recall that in the $\delta$-HMX crystals, the free surfaces, vacancies, and voids are charged and hence contribute to electric conductivity while, in the nonpolar $\beta$-HMX crystals, the defects are neutral and do not increase conductivity, the difference in activation energies 
becomes logical and anticipated. Note that the measurements reflect combined surface and bulk conductivity $\mathrm{n}$ the samples $\left[{ }^{54}\right]$.

We predict that the surface conductivity in HMX polymorphs will have even more dramatic difference as the charge density corresponding surface charge density is $7.9^{*} 10^{13}$ $\mathrm{e} / \mathrm{cm}^{2}$, which is similar to a typical value of the charge carrier density at the oxide/oxide interfaces (for example, $2 * 10^{13} \mathrm{e} / \mathrm{cm}^{2}$ is reported for LAO/STO interface $\left[{ }^{53}\right]$ ). 
ס-HMX
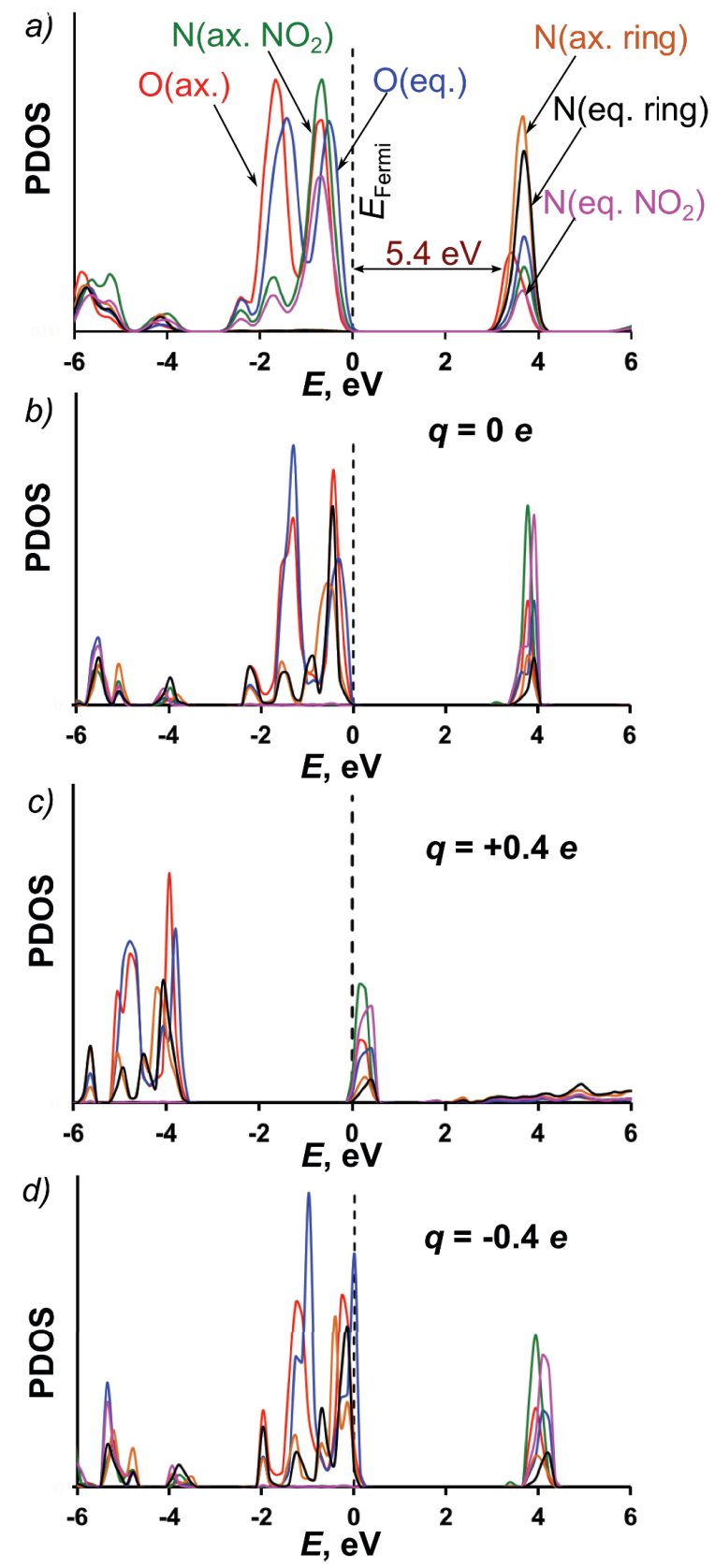

$\beta-H M X$
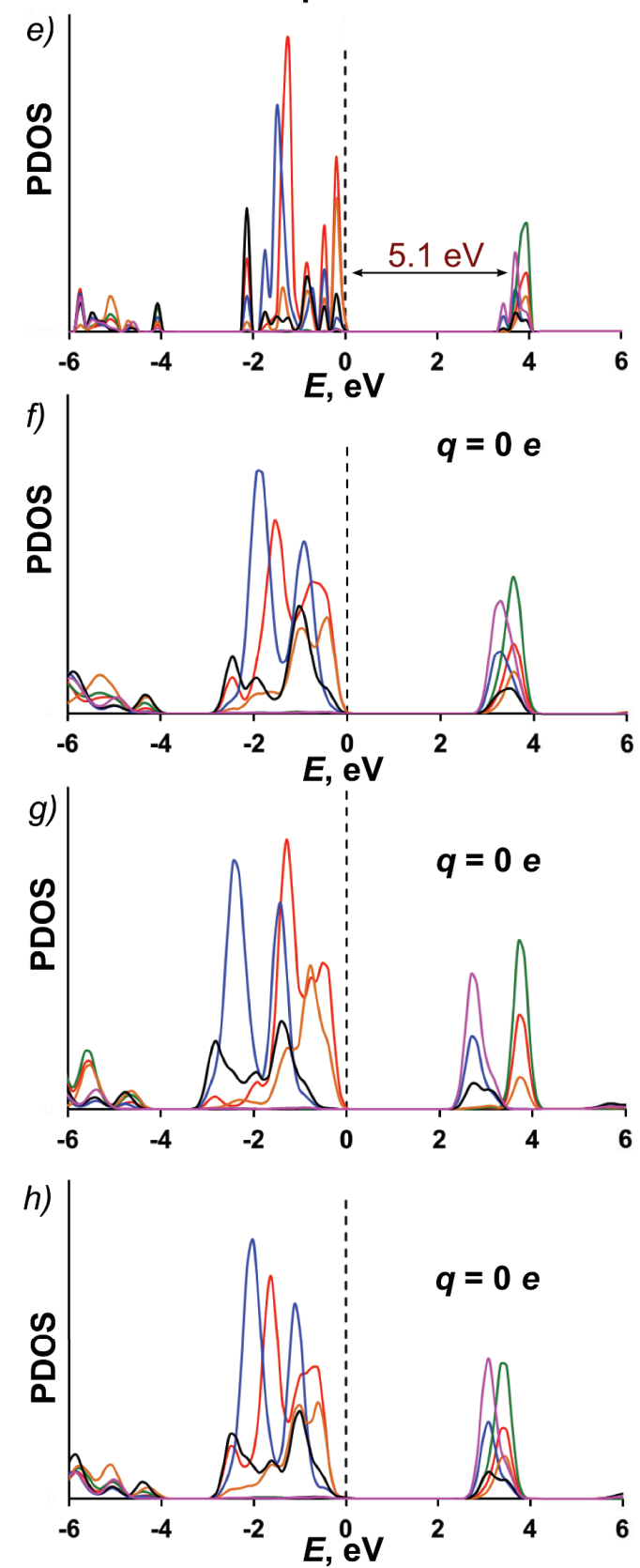

Figure 4. DOS (in arbitrary units) projected to the $\delta$-HMX molecules placed at a) the ideal bulk, b) the middle layer of the surface slab, c) positively charged surface, and d) negatively charged surface of the $\delta$-HMX crystal; and projected to the $\beta$-HMX molecules placed at e) the ideal bulk, f) the middle layer of the surface slab, $\mathbf{g - h}$ ) outer layers of (100) surface slab of the $\beta$-HMX crystal. PDOS was obtained with PBE functional, and the band gaps were refined with HSE06 functional. The figures show that $\beta$-HMX is insulating both on the surface and bulk layers while $\delta$-HMX is insulating in the bulk layer $(\mathrm{a}, \mathrm{b})$ and conducting in the surface layers ( $c$ and $d$ ). Conductivity of the surface layers can be seen from the position of the Fermi level relative to the valence and the conduction bands. 
Further, to explore the optical absorption properties of the molecule, ideal bulk and (100) surface of $\delta$-HMX, the frequency-dependent imaginary part of the dielectric function (Fig. 5a) was calculated. Figure 5a shows that the molecule and ideal bulk begin absorbing light at the energies above $\sim 4.5 \mathrm{eV}$, with two maximums observed at $\sim 6$ and $6.7 \mathrm{eV}$. This is consistent with conductivity measurements $\left[{ }^{54}\right]$ and fully agrees with the results obtained from the gasphase calculations carried out for a single $\delta$-HMX molecule using TD HSE06 functional. The energy of the lowest singlet-singlet transition is predicted to be $4.75 \mathrm{eV}$, and the absorption maximum corresponds to the singlet-singlet transition with the energy of $5.88 \mathrm{eV}$.

Figure $5 \mathrm{~b}$ shows that both the isolated molecule and ideal bulk of $\beta$-HMX exhibit optical properties similar to $\delta$-HMX. The calculated energy of the lowest singlet-singlet transition (obtained from TD HSE06 functional) of the $\beta$-HMX molecule is $4.76 \mathrm{eV}$ and the absorption maximum corresponds to the singlet-singlet transition with the energy of $6 \mathrm{eV}$.

Figure 5a clearly indicates that the polar (001) $\delta$-HMX surface absorbs light, similarly to the molecule and ideal bulk, in the energy range of $4.5-8 \mathrm{eV}$, with the pronounced maximum at $6.7 \mathrm{eV}$ and somewhat smeared maximum at $\sim 6 \mathrm{eV}$. In addition, a new distinct low energy absorption band appears at the range of $0-1 \mathrm{eV}$ in Fig. 5a. This band is attributed to the $\delta$ HMX surface and does not occur in either the molecular or bulk crystal absorption spectra. Figure $5 \mathrm{~b}$ reveals that (100) surface of $\beta$-HMX absorbs light with the energy $>4 \mathrm{eV}$ similar to ideal bulk and gas-phase molecule, whereas no evidence of low energy transition in the range $0-1 \mathrm{eV}$ was found. A comparison of the optical properties of $\beta$ - and $\delta$-phases implies that the high quality $\beta$-HMX samples are likely to be in the form of transparent or white crystals that will change its color to opaque dark while transforming into $\delta$-HMX. 

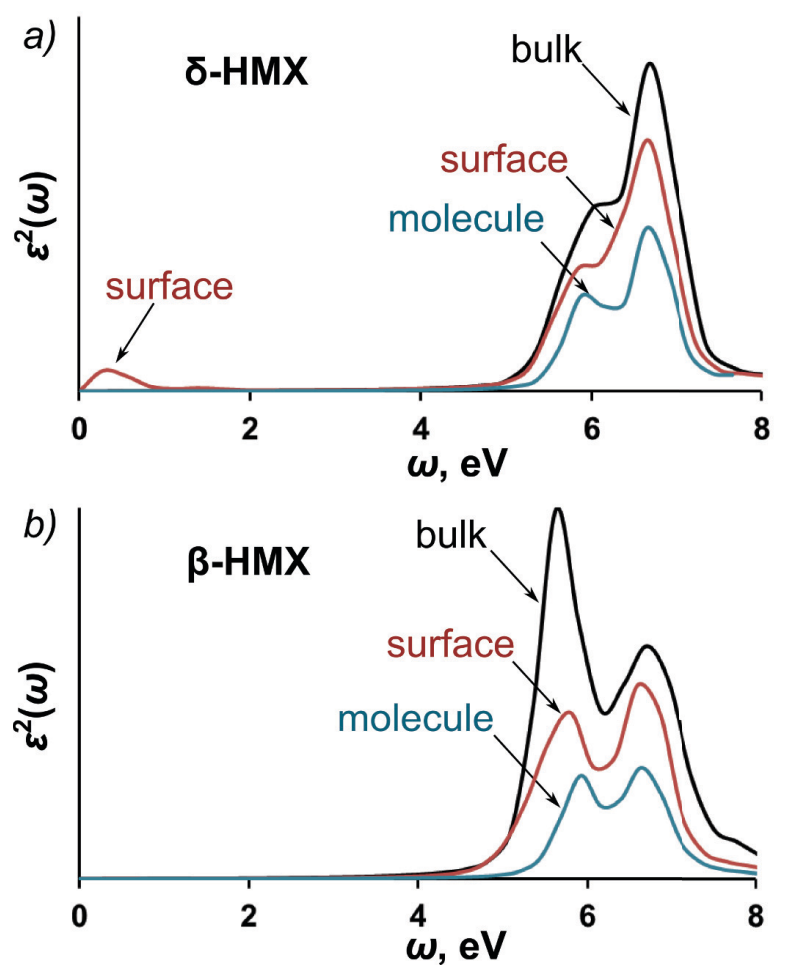

Figure 5. The frequency-dependent imaginary part of the dielectric function of the a) $\delta$-HMX molecule, ideal bulk crystal, and (001) surface; b) $\beta$-HMX molecule, ideal bulk crystal, and (100) surface is calculated with HSE06 functional. Clearly, there are no local electronic states in the gap of the bulk $\delta$-HMX, and the small absorption peak in Figure a) near the Fermi level is associated with the surface states.

Furthermore, peculiarities of the surface stability of polar $\delta$-HMX and non-polar $\beta$-HMX polymorphs were recently analyzed in great detail $\left[{ }^{26,27}\right]$. While the homolytic cleavage of the $\mathrm{N}-\mathrm{N}$ bond was found to be predominant pathway for thermal decomposition of both $\delta$ - and $\beta$ HMX polymorphs $\left[{ }^{26,47}\right]$, the significantly lower activation energy $(\sim 20 \mathrm{kcal} / \mathrm{mol})$ is required to breakdown the (001) surface $\delta$-HMX than the $\beta$-HMX surface $(\sim 40 \mathrm{kcal} / \mathrm{mol}$, Table 1$)$. The decomposition mechanisms that represent a complex multi-stage process proceeding via the formation and isomerization of aci-isomers of $\delta$-HMX and the corresponding activation barriers of the charged (001) $\delta$-HMX surfaces are consistent with those of $\delta$-HMX anion and cation radicals $\left[{ }^{26}\right]$. In contrast, the decomposition of $\delta$-HMX gas-phase molecules and ideal bulk is not much different from the bond breaking of non-polar $\beta$-HMX $\left[{ }^{27}\right]$ (Table 1). 
Table 1. Energies (kcal/mol) of the N-N bond scission in molecules, ideal bulk crystals, and surfaces, as well as band gaps are shown for $\beta$ - and $\delta$-HMX.

\begin{tabular}{|c|c|c|c|c|}
\hline \multirow{2}{*}{ HMX } & \multicolumn{2}{|c|}{$\mathbf{E}_{\text {barrier }}(\mathrm{kcal} / \mathrm{mol})$} & \multicolumn{2}{c|}{ Band Gap (eV) } \\
\cline { 2 - 5 } & $\boldsymbol{\beta}$-HMX & $\boldsymbol{\delta}$-HMX & $\boldsymbol{\beta}$-HMX & $\boldsymbol{\delta}$-HMX \\
\hline \hline Isolated molecule & 44.8 & 45.1 & 5.53 & 6.0 \\
\hline Ideal bulk crystal $^{\text {a }}$ & 47.9 & 50.8 & 5.10 & 5.45 \\
\hline $\begin{array}{c}\text { Surface }^{\mathrm{a}} \\
\text { charge] }^{\mathrm{b}}\end{array}$ & $40.1[0]$ & $\begin{array}{c}20.7[+] \\
20.1[-]\end{array}$ & 4.70 & 0 \\
\hline \hline
\end{tabular}

${ }^{a}$ Energies of the N-N bond scission and values of Band Gaps are depicted for the (100) surface of $\beta$-HMX and (001) surface of $\delta$-HMX

${ }^{\mathrm{b}}$ Surface charges are shown in brackets

Despite the abundance of data on thermal decomposition of HMX $\left[{ }^{55,56,57}\right]$ precise measurements that would distinguish the dissociation pathways and allow us compare the predicted $\beta$ - and $\delta$-HMX surface degradation processes are not available for the time being. Nevertheless, the observation that $\delta$-HMX samples exhibit appreciably faster decomposition kinetics $\left[{ }^{55,56,57}\right]$ and therefore higher thermal sensitivity to detonation initiation is consistent with our conclusions. Our results, in fact, provide a plausible explanation to those experiments.

\section{IV.Discusssion}

Our findings are consistent with observations by other researchers. Accelerated surface chemistry was reported for other polar molecular crystals. An example is the reaction with ammonia of p-chlorobenzoic anhydride to give a microcrystalline conglomerate of $\mathrm{p}$ chlorobenzamide and ammonium p-chlorobenzoate $\left[{ }^{58}\right]$. Curtin and Paul suggested that a puzzling difference in the anisotropy of the reactions of the polar bromo and nonpolar chloro anhydrides is that, while the chloro anhydride undergoes reaction at approximately the same rate in any direction parallel to the major face of the crystal, the bromo anhydride reacts much faster along the polar axis than along those directions normal to it $\left[^{53}\right]$. Curtin and Paul thought that the similarity in packing of the centrosymmetric p-chlorobenzoic anhydride to the noncentrosymmetric p-bromo compound suggests that it might be possible to induce the pchloro anhydride to crystallize with a polar structure by application of an electric field during the crystallization process. We propose that the same notion can be applied to enhance or reduce (i.e. to control) the amount of polar phase molecules versus nonpolar phase molecules 
in various polymorphs, and thus to tune sensitivity to initiation of chemistry, i.e. to accelerate or suppress surface degradation on demand.

Using the example of oligopyrimidinethiols, Rissner et al $\left[{ }^{59}\right]$ have demonstrated that in self-assembled monolayers (SAM) of polar molecules, the band gap rapidly decreases to zero at a certain molecular length of repeated units and the frontier states localize on opposite ends of the monolayer. This is accompanied by a saturation of the dipole moment per molecule at the same number of repeat units at which the gap closes. The authors emphasize that this effect is not observed in the isolated polar molecules, but only in periodic systems $\left[{ }^{59}\right]$.

Finally, it is essential to emphasize the difference between molecular crystals and oxides because polarization-induced effects in these classes of solids are manifested in unalike ways. On metal oxide surfaces, it is shown that the polar catastrophe conditions are eliminated due to the band bending through the internal electric field caused by the polar surfaces $\left[{ }^{32,33,34,35}\right]$. This, in turn, leads to charge transfer between the two surfaces, and results in a finite, not a divergent contribution to the surface energy $\left.{ }^{32,36,37,38}\right]$. Hence all crystals with polar surfaces should exhibit some excess surface charge. Therefore, it is demonstrated that the polar catastrophe is not the main mechanism of $2 \mathrm{D}$ electron gas, but it is a combination of surface and interface defects that compensate for polarization $\left[{ }^{32,60}\right]$. In metal oxides, the polar layer is created between an oxide and a metal. Point defects in metal oxides are charged and therefore vacancies or/and interstitials ideally serve to completely cancel the dipole field.

Here, we suggest that in molecular crystals, the most feasible compensation mechanism is the charge transfer from the valence to the conduction band due to the band bending. Alternatively, the divergent field can be eliminated by surface defects and a surface reconstruction. In molecular crystals, the dipole moment exists within a single molecule. On the other hand, molecular vacancies are neutral and hence they do not change polarization of the crystal. A removal of an entire molecule removes both the positive and negative charge and the net polarization remains the same. Hence, unlike on polar oxide surfaces or interfaces, vacancies on molecular crystals do not provide a compensation mechanism to stabilize surfaces. Because of this difference, in molecular crystals, the electric field-induced surface charge is a plausible mechanism of compensation for polar discontinuity.

Also, an important difference between oxides and molecular crystals is that the charge and dipole of a molecule can be uniquely defined for a molecule in the crystal (because of 
this, equation 3 is accurate for molecular crystals). In oxides, the surface charge depends on the choice of the unit cell, in molecular crystals, it does not.

\section{Conclusions}

Summing up, we explored the polar catastrophe phenomenon in molecular materials with an illustrating example of the organic crystal of HMX. We showed analytically that the polar discontinuity is inevitable unless molecules on the surface layer are charged. Using first principles calculations we found that the band gap of the surface layer of the dielectric molecular crystal becomes zero and that electronic states of the top of the valence band and of the bottom of the conduction band localize on opposite ends of the model surface slab. The seeming polar catastrophe manifests in significantly different surface behavior of polar and nonpolar polymorphs of materials with similar stoichiometry but different crystalline symmetries.

We expect that the similar manifestations of polar discontinuity occur on most polar organic crystals although other polar molecular materials may reveal the effect in a variety of ways.

We would like to emphasize the main difference between polar organic crystals and polar metal oxides. While the dipole in oxides is created between cation and anion layers, the dipole in polar molecular crystals is formed within a single molecule. This difference makes the polar catastrophe most plausible mechanism to compensate for the polarization discontinuity. A recent analysis $\left[{ }^{60}\right]$ showed that the polar catastrophe cannot explain a wide range of phenomena in oxides. On the other hand, polar molecular crystals, including HMX, can serve as good examples where this phenomenon is demonstrated. The described polar instability phenomena in molecular materials are fundamentally important for both basic science and technology applications. Potentially, it may open new perspectives in such fields as surface science, heterogeneous catalysis, energy storage and conversion, self-assembly and synthesis of materials, design of new materials for optoelectronic devices (that e.g. facilitate the dissociation of excitons in solar cells), sensors and detectors, and controllable surface behavior of materials interfaces.

\section{ACKNOWLEDGMENTS}


This research is supported in part by ONR (Grants N00014-12-1-0529, N00014-16-1-2069, and N00014-16-1-2346) and NSF. We used NSF XSEDE resources (Grant DMR-130077), DOE NERSC resources (Contract DE-AC02-05CH11231), and computational resources at the Maryland Advanced Research Computing Center (MARCC). MMK is grateful to the Office of the Director of National Science Foundation for support under the IRD program. Any appearance of findings, conclusions, or recommendations expressed in this material are those of the authors and do not necessarily reflect the views of NSF. 


\section{References and notes}

$\left.{ }^{1}\right]$ A. Ohtomo, and H. Y. Hwang, A high-mobility electron gas at the LaAlO3/SrTiO3 heterointerface, Nature 163 (2004) 423-426.

$\left.{ }^{2}\right]$ K. v. Klitzing, G. Dorda, M. Pepper, New Method for High-Accuracy Determination of the Fine-Structure Constant Based on Quantized Hall Resistance, Phys. Rev. Lett. 45 (1980) 494497.

$\left[{ }^{3}\right]$ S. Okamoto, and A. J. Millis, Electronic reconstruction at an interface between a Mott insulator and a band insulator, Nature 428 (2004) 630-633.

[ $\left.{ }^{4}\right]$ C. H. Ahn, J.-M. Triscone, J. Mannhart, Electric field effect in correlated oxide systems, Nature 424 (2003) 1015-1018.

$\left.{ }^{5}\right]$ A. Ohtomo, D. A. Muller, J. L. Grazul, H. Y. Hwang, Artificial charge-modulation in atomic-scale perovskite titanate superlattices, Nature 419 (2002) 378-380.

[6] C. Cen, S. Thiel, G. Hammer, C. W. Schneider, K. E. Andersen, C. S. Hellberg, J. Mannhart, J. Levy, Nanoscale control of an interfacial metal-insulator transition at room temperature, Nature Mater. 7 (2008) 298-302.

[7] S. Thiel, G. Hammer, A. Schmehl, C. W. Schneider, J. Mannhart, Tunable quasi-twodimensional electron gases in oxide heterostructures, Science 313 (2006) 1942-1945.

$\left[{ }^{8}\right]$ N. Reyren, S. Thiel, A. D. Caviglia, L. Fitting Kourkoutis, G. Hammer, C. Richter, C. W. Schneider, T. Kopp, A.-S. Rüetschi, D. Jaccard, M. Gabay, D. A. Muller, J.-M. Triscone1, J. Mannhart, Superconducting interfaces between insulating oxides. Science 317 (2007) 11961199.

[9] K. Ueno, S. Nakamura, H. Shimotani, A. Ohtomo, N. Kimura, T. Nojima, H. Aoki, Y. Iwasa, M. Kawasaki, Electric-field-induced superconductivity in an insulator, Nature Mater. 7 (2008) 855-858.

$\left.{ }^{10}\right]$ A. D. Caviglia, S. Gariglio, N. Reyren, D. Jaccard, T. Schneider, M. Gabay, S. Thiel, G. Hammerl, J. Mannhart, J.-M. Triscone, Electric field control of the $\mathrm{LaAlO}_{3} / \mathrm{SrTiO}_{3}$ interface ground state, Nature 456 (2008) 624-627. 
$\left.{ }^{11}\right]$ A. Brinkman, M. Huijben, M. van Zalk, J. Huijben, U. Zeitler, J. C. Maan, W. G. van der Wiel, G. Rijnders, D. H. A. Blank, H. Hilgenkamp, Magnetic effects at the interface between non-magnetic oxides, Nature Mater. 6 (2007) 493-496.

$\left.{ }^{12}\right]$ L. Li, C. Richter, J. Mannhart, R. C. Ashoori, Coexistence of magnetic order and twodimensional superconductivity at $\mathrm{LaAlO}_{3} / \mathrm{SrTiO}_{3}$ interfaces, Nat. Phys. 7 (2011) 762-766.

$\left[{ }^{13}\right]$ H. Ohta, S.W. Kim, Y. Mune, T. Mizoguchi, K. Nomura, S. Ohta, T. Nomura, Y. Nakanishi, Y. Ikuhara, M. Hirano, H. Hosono, K. Koumoto, Giant thermoelectric Seebeck coefficient of a two-dimensional electron gas in $\mathrm{SrTiO}_{3}$, Nature materials 6 (2007) 129-134.

$\left[{ }^{14}\right]$ A. P. Ramirez, Oxide electronics emerge, Science 315 (2007) 1377-1378.

$\left[{ }^{15}\right]$ C. Cen, S. Thiel, J. Mannhart, J. Levy, Oxide nanoelectronics on demand, Science 323 (2009) 1026-1030.

$\left[{ }^{16}\right]$ Frisch, M. J. et al. Gaussian 09, revision B.01; Gaussian, Inc.: Wallingford, CT, 2010.

$\left[{ }^{17}\right]$ P. Hohenberg, W. Kohn, Inhomogeneous Electron Gas, Phys. Rev. B 136 (1964) 864-871.

$\left[{ }^{18}\right]$ W. Kohn, L. J. Sham, Self-consistent Equations Including Exchange and Correlation Effects, Phys. Rev. A 140 (1965) 1133-1138.

$\left[{ }^{19}\right]$ J. Heyd, G. Scuseria, Efficient Hybrid Density Functional Calculations in Solids: Assessment of the Heyd-Scuseria-Ernzerhof Screened Coulomb Hybrid Functional, J. Chem. Phys. 121 (2004) 1187-92.

$\left[{ }^{20}\right]$ R. Bauernschmitt, R. Ahlrichs, Treatment of Electronic Excitations within the Adiabatic Approximation of Time Dependent Density Functional Theory, Chem. Phys. Lett. 256 (1996) 454-64.

$\left[{ }^{21}\right]$ M. E. Casida, C. Jamorski, K. C. Casida, D. R. Salahub, Molecular Excitation Energies to High-lying Bound States from Time-dependent Density-functional Response Theory: Characterization and Correction of the Time-dependent Local Density Approximation Ionization Threshold, J. Chem. Phys. 108 (1998) 4439-4449.

$\left[{ }^{22}\right]$ P. E. Blöchl, Projector augmented-wave method, Phys. Rev. B 50 (1994) 17953-17979.

$\left[{ }^{23}\right]$ G. Kresese, J. Futhmuller, Efficiency of ab-initio total energy calculations for metals and semiconductors using a plane-wave basis set, Comput. Mater. Sci. 6 (1996) 15-50.

$\left[{ }^{24}\right]$ G. Kresse, J. Furthmuller, Efficient iterative schemes for ab initio total-energy calculations using a plane-wave basis set, Phys. Rev. B 54 (1996) 11169-11186. 
$\left[{ }^{25}\right]$ G. Kresse, J. Hafner, Ab initio molecular dynamics for liquid metals, Phys. Rev. B47 (1993) RC558-561.

$\left.{ }^{26}\right]$ M. Kuklja, R. Tsyshevsky, O. Sharia, Effect of polar surfaces on decomposition of molecular materials, J. Am. Chem. Soc. 136 (2014) 13289-13302.

$\left[{ }^{27}\right]$ O. Sharia, M. M. Kuklja, Rapid materials degradation induced by surfaces and voids: ab initio modeling of $\beta$-octatetramethylene tetranitramine, J. Am. Chem. Soc. 134 (2012) 1181511820 .

$\left[{ }^{28}\right]$ M. Gajdoš, K. Hummer, G. Kresse, J. Furthmüller, F. Bechstedt, Linear optical properties in the PAW methodology, Phys. Rev. B73 (2006) 045112.

$\left.{ }^{29}\right]$ W. Tang, E. Sanville, G. Henkelman, A grid-based Bader analysis algorithm without lattice bias, J. Phys.: Condens. Matter 21 (2009) 084204-1-7.

$\left.{ }^{30}\right]$ E. Sanville, S. D. Kenny, R. Smith, G. Henkelman, Improved grid-based algorithm for Bader charge allocation, J. Comp. Chem. 28 (2007) 899-908.

$\left.{ }^{31}\right]$ G. Henkelman, A. Arnaldsson, H. Jónsson, A fast and robust algorithm for Bader decomposition of charge density, Comput. Mater. Sci. 36 (2006) 254-360.

$\left[{ }^{32}\right]$ C. Noguera, Polar oxide surfaces, J. Phys. Condens. Matter 12 (2000) R367-R410.

$\left[{ }^{33}\right]$ C. Noguera, J. Goniakowski, Polarity of oxide surfaces and nanostructures, J. Phys.: Condens. Matter 20, (2008) 264003-1-10.

$\left[{ }^{34}\right]$ N.G. Hörmann, A. Groß, Polar Surface Energies of Iono-Covalent Materials: Implications of a Charge-Transfer Model Tested on $\mathrm{Li}_{2} \mathrm{FeSiO}_{4}$ Surfaces, Chem. Phys. Chem. 15 (2014) $2058-2069$.

$\left[{ }^{35}\right]$ A. Eichler, G. Kresse, First-principles calculations for the surface termination of pure and yttria-doped zirconia surfaces, Phys. Rev. B 69 (2004) 045402-1-17.

$\left[{ }^{36}\right]$ A. Wander, F. Schedin, P. Steadman, A. Norris, R. McGrath, T. S. Turner, G. Thornton, N. M. Harrison, Stability of Polar Oxide Surfaces, Phys. Rev. Lett. 86 (2001) 3811-3814.

$\left[{ }^{37}\right]$ Noguera, C. Physics and Chemistry at Oxide Surfaces. Cambridge University Press, Cambridge, 1996.

$\left[{ }^{38}\right]$ J. Goniakowski, F. Finocchi, C. Noguera, Polarity of oxide surfaces and nanostructures, Rep. Prog. Phys. 71 (2008) 016501-1-55. 
$\left[{ }^{39}\right]$ C. S. Choi, H. P. Boutin, A study of the crystal structure of $\beta$-cyclotetramethylene tetranitramine by neutron diffraction, Acta Crystallogr. B26 (1970) 1235-1240.

$\left[{ }^{40}\right]$ R.E. Cobbledick, R.W.H. Small, The crystal structure of the $\delta$-form of 1,3,5,7-tetranitro1,3,5,7-tetraazacyclooctane ( $\delta$-HMX), Acta Crystallogr. B30 (1974) 1918-1922.

$\left[{ }^{41}\right]$ T. B. Brill, R. J. Karpowicz, Solid phase transition kinetics. The role of intermolecular forces in the condensed-phase decomposition of octahydro-1, 3, 5, 7-tetranitro-1, 3, 5, 7tetrazocine, J. Phys. Chem. 86 (1982) 4260-4265.

$\left.{ }^{42}\right]$ G. Wulff, Zur Frage der Geschwindigkeit des Wachstums und der Auflösung der Krystallflachen, Zeitschr. Krystallogr. Mineral. 34 (1901) 449-530.

$\left[{ }^{43}\right]$ A. Dinghas, Uber einen geometrischen Satz von Wulff für die Gleichtgewichts-form von Kristallen, Zeitschr. Kristallogr. Mineral. 105 (1944) 301-314.

$\left[{ }^{44}\right]$ C. Herring, Some Theorems on the Free Energies of Crystal Surfaces, Phys. Rev. 82 (1951) 87-93.

$\left[{ }^{45}\right]$ R. Dobrushin, R. Kotecký, S. Shlosman. Wulff construction: a global shape from local interaction. American Mathematical Society, Providence, Rhode Island, 1992.

$\left[{ }^{46}\right]$ B. F. Henson, B. W. Asay, R. K. Sander, S. F. Son, J. M. Robinson, P. M. Dickson, Dynamic measurement of the HMX $\beta-\delta$ Phase transition by second harmonic generation, Phys. Rev. Lett. 82 (1999) 1213-1216.

$\left[{ }^{47}\right]$ O. Sharia, R. Tsyshevsky, M. M. Kuklja, Surface-Accelerated Decomposition of $\delta$-HMX, J. Phys. Chem. Lett. 4 (2013) 730-734.

$\left[{ }^{48}\right]$ Jackson, J. D. Classical Electrodynamics. Wiley, NY, 1998.

$\left[{ }^{49}\right]$ R. D. King-Smith, D. Vanderbilt, Theory of polarization of crystalline solids, Phys. Rev. B 47 (1993) 1651-1654.

$\left.{ }^{50}\right]$ R. Resta, Macroscopic polarization in crystalline dielectrics: the geometric phase approach, Rev. Mod. Phys. 66 (1994) 899-915.

$\left.{ }^{51}\right]$ R. Resta, D. Vanderbilt, Physics of Ferroelectrics: A Modern Perspective. In Topics of Applied Physics, Springer, Berlin 31-68, 2007.

$\left[{ }^{52}\right]$ An ambiguity in defining polarization and surface charges in ionic crystals, which leads to an uncertainty of the unit cell choice, was addressed by Born effective charges introduced by 
Vanderbilt, see ref. 49-51. Due to their structural nature, molecular crystals have an advantage and do not require this treatment.

$\left[{ }^{53}\right]$ J. Lee, A. A. Demkov, Charge origin and localization at the n-type $\mathrm{SrTiO}_{3} / \mathrm{LaAlO}_{3}$ interface, Phys. Rev. B 78 (2008) 193104.

$\left[{ }^{54}\right]$ J. N. Maycock, V. R Pai Verneker, L. L. Rouch Jr., Physico-Chemical Properties of 1,3,5,7-Tetranitro-1,3,5,7-Tetrazacyclooctane(HMX), Phys. Stat. Sol. B35 (1969) 843-847.

$\left[{ }^{55}\right]$ C. M. Tarver, T. D. Tran, Thermal decomposition models for HMX-based plastic bonded explosives, Combustion and Flame 137 (2004) 50-62.

$\left[{ }^{56}\right]$ D. J. Whelan, R. F. Mark, A Reassessment of the Kinetics of the Thermal Decomposition of the High Explosive, Delta-HMX, in the Range $508 \mathrm{~K}$ to $524 \mathrm{~K}$, as Studied by Isothermal Gravimetry. NASA 19980218798 (1998)

$\left[{ }^{57}\right]$ S. Bulusu, D. I. Weinstein, J. R. Autera, R. W. Velicky, Deuterium kinetic isotope effect in the thermal decomposition of 1,3,5-trinitro-1,3,5-triazacyclohexane and 1,3,5,7-tetranitro1,3,5,7-tetraazacyclooctane: its use as an experimental probe for their shock-induced chemistry, J. Phys. Chem. 90 (1986) 4121-4126.

$\left[{ }^{58}\right]$ D. Y. Curtin, I. C. Paul, Chemical consequences of the polar axis in organic solid-state chemistry, Chem. Rev. 81 (1981) 528-541.

$\left.{ }^{59}\right]$ F. Rissner, A. Natan, D. A. Egger, O. T. Hofmann, L. Kronik, E. Zojer, Dimensionality effects in the electronic structure of organic semiconductors consisting of polar repeat units, Organic Electronics 13 (2012) 3165-3176.

$\left[{ }^{60}\right]$ L. Yu, A. Zunger, A polarity-induced defect mechanism for conductivity and magnetism at polar-nonpolar oxide interfaces, Nature Communications 5 (2014) 1-9. 